\title{
Insulin-like growth factor-1 effects on bovine retinal endothelial cell glucose transport: role of MAP kinase
}

\author{
Brian J. DeBosch, ${ }^{*}$ Baljit K. Deo* and Arno K. Kumagai, ${ }^{*} \uparrow \ddagger$ \\ *Department of Internal Medicine, †Michigan Diabetes Research \& Training Center and $\ddagger J D R F$ Center for Complications in \\ Diabetes, University of Michigan Medical School, Ann Arbor, Michigan, USA
}

\begin{abstract}
In order to maintain normal metabolism, the neuroretina is completely dependent on the constant delivery of glucose across the retinal microvascular endothelial cells comprising the inner blood-retinal barrier. Glucose uptake into these cells is influenced by various stimuli, including hypoxia and growth factors. Recently, insulin-like growth factor-1 (IGF-1) was shown to enhance retinal endothelial glucose transport in a process that is dependent on protein kinase $C$ (PKC) and phosphatidylinositol-3 kinase (PI3 kinase). In the current study, the role of mitogen-activated protein kinase (MAP kinase) in regulating IGF-1 effects on retinal endothelial cell glucose transport was investigated in a bovine retinal endothelial cell (BREC) culture model. IGF-1 (25 ng/mL) caused a rapid increase in MAP-kinase activity and ERK phosphorylation. Inhibition of MAP kinase with PD98059 (100 $\mu \mathrm{m})$ blocked IGF-1 enhancement of 2-deoxyglucose uptake. In order to
\end{abstract}

clarify the relationship between PKC, PI3 kinase and MAP kinase in IGF-1 signaling in retinal endothelial cells, the effects of selective inhibitors of MAP kinase (PD98059), PKC (GF109203X), and PI3 kinase (wortmannin, LY294002) on signal transduction by IGF-1 were studied. Inhibition of MAP kinase abolished IGF-1 stimulation of PKC but had no effect on PI3 kinase activity, whereas inhibition of either PKC and PI3 kinase had no effect on MAP kinase phosphorylation or activity in IGF-1-treated cells. Taken together, these data demonstrate that IGF-1 stimulation of BREC glucose transport requires activation of MAP kinase and that MAP kinase is upstream from PKC but is independent of PI3 kinase in mediating the actions of IGF-1 on retinal endothelial cells.

Keywords: diabetic retinopathy, glucose transport, GLUT1, inner blood-retinal barrier, insulin-like growth factor, retinal endothelial cells.

J. Neurochem. (2002) 81, 728-734.
Under normal physiological conditions, the retina is completely dependent on glucose for normal metabolism, and because the retina contains minimal stores of glycogen in comparison to metabolic demand, both the developing and mature retina are completely dependent on the continuous delivery of glucose from the systemic circulation. Delivery of glucose into the neuroretina involves its transport across the specialized retinal microvascular endothelial cells that comprise the so-called inner blood-retinal barrier (inner BRB) (Cunha-Vaz 1976) and occurs via a facilitative transport process mediated by the sodium-independent GLUT1 glucose transporter (Takata et al. 1997; Kumagai 1999). The neuronal glucose transporter, GLUT3, has also been reported to be expressed in human retinal endothelial cells in culture (Knott et al. 1996); however, interpretation of this finding is difficult in light of reports of an absence of GLUT3 in the plasma membrane of bovine retinal endothelial cell (BREC) cultures (Mandarino et al. 1994), and more importantly of an absence of GLUT3 in the microvascular endothelial cells of the human retina (Mantych et al. 1993).

GLUT1-mediated transport into retinal endothelial cells is regulated by hypoxia (Takagi et al. 1998) and various growth factors (Sone et al. 2000; DeBosch et al. 2001). We have recently shown that vascular endothelial cell growth factor (VEGF) and insulin-like growth factor-1 (IGF-1), peptides

Received December 12, 2001; revised manuscript received January 24, 2002; accepted January 28, 2002.

Address correspondence and reprint requests to Arno K. Kumagai, MD, Department of Internal Medicine, 5570 MSRB-2, University of Michigan Medical School, Ann Arbor, MI 48109-0678, USA.

E-mail: akumagai@umich.edu

Abbreviations used: BREC, bovine retinal endothelial cells; 2DG, 2-deoxyglucose; DMEM, Dulbecco's modified Eagle's medium; EGS, endothelial growth supplement; FCS, fetal calf serum; IGF-1, insulinlike growth factor-1; MAP, kinase, mitogen-activated protein kinase; $\mathrm{PKC}$, protein kinase C; PI3, kinase, phosphatidylinositol-3 kinase; VEGF, vascular endothelial cell growth factor. 
that act as potent endothelial cell mitogens in retinal development and in pathophysiological states such as diabetic retinopathy (Robinson and Aiello 1998), enhance glucose transport in primary cultures of BREC (Sone et al. 2000; DeBosch et al. 2001). This stimulation of glucose uptake by both growth factors occurs in a dose-dependent manner through a process that involves activation of protein kinase C- $\beta$ (PKC- $\beta$ ) (Sone et al. 2000; DeBosch et al. 2001). In the case of IGF-1, experiments using pharmacological inhibitors of PKC and phosphatidylinositol-3 kinase (PI3 kinase) have demonstrated that IGF-1-mediated stimulation of glucose transport requires activation of both $\mathrm{PKC}$ and PI3 kinase and that IGF-1 activation of PKC was preceded by, and dependent upon, PI3 kinase activation in this cell culture model (DeBosch et al. 2001). It is currently not known if other signal transduction pathways that participate in vital cellular processes in growth and development also participate in IGF-1 enhancement of retinal endothelial cell glucose transport.

The mitogen-activated protein kinases (MAP kinases) consist of a series of protein kinases that link signals at the plasma membrane with nuclear events. These kinases are activated by a variety of extracellular stimuli via dual phosphorylation on highly conserved threonine and tyrosine residues (Seger and Krebs 1995; English et al. 1999). Three major pathways have been described: the extracellular-signal regulated kinases (ERK1/ERK2, also known as the p42/p44 MAP kinases), the c-Jun N-terminal kinases (JNK, also known as the stress-activated protein kinases, or SAPK), and p38 kinase. Of the various pathways, the ERK1/ERK2 pathway (hereafter referred to as the MAP kinase pathway) has been most extensively characterized and has been shown to be essential in processes involving cellular proliferation and differentiation both in vitro and in vivo (Seger and Krebs 1995; English et al. 1999). Activation of this pathway by various growth factors involves sequential participation of Ras-GTP, Raf, MEK1/MEK2 (also known as MAP kinase kinase-1 and 2) and ERK1 and ERK2. Phosphorylation of ERK1 and 2 may stimulate their translocation to the nucleus, where they may in turn phosphorylate a variety of substrates, including ELK1, c-Jun, c-Fos and ATF2, among others, which may play a role in involved in cellular proliferation, growth and differentiation (English et al. 1999).

The objectives of the current study were three-fold: first, to characterize activation of the MAP kinase pathway by IGF-1 in primary retinal endothelial cells; second, to determine whether activation of this pathway is required for IGF-1mediated BREC glucose transport; and third, to characterize the relationship of MAP kinase signaling by IGF-1 in relationship to two other signal transduction intermediates regulating actions of this growth factor in retinal endothelial cells, PKC and PI3 kinase. We report that MAP kinase, like $\mathrm{PKC}$ and $\mathrm{PI} 3$ kinase, is required for enhancement of glucose transport by IGF-1, and that activation of the MAP kinase pathway is necessary for IGF-1 stimulation of PKC, but is independent of PI3 kinase activation by IGF-1 in retinal endothelial cells.

\section{Materials and methods}

\section{Reagents}

Dulbecco's modified Eagle's medium (DMEM) and endothelial growth supplement (EGS) used in the BREC cultures were purchased from Gibco-BRL (Grand Island, NY, USA) and Boehringer Mannheim (Indianapolis, IN, USA), respectively. PD98059 and LY294002 were purchased from Calbiochem (San Diego, CA, USA). GF109203X, wortmannin, cytochalasin-B and phloretin were obtained from Sigma (St Louis, MO, USA). The glucose analog, 2-[1,2- $\left.{ }^{3} \mathrm{H}(N)\right]$-deoxy-D-glucose $\left(\left[{ }^{3} \mathrm{H}\right] 2 \mathrm{DG}\right)$ and the ECL-Plus Chemiluminescence kit were from Amersham (Piscataway, NJ, USA). The anti-phospho specific and anti-total ERK antisera were purchased from Cell Signaling Technologies (Beverly, MA, USA) and Santa Cruz Biochemicals (Santa Cruz, CA, USA), respectively. The $\left[\gamma-{ }^{32} \mathrm{P}\right] \mathrm{ATP}$ used in the PKC activity and PI3 kinase assays was purchased from Dupont-NEN (Boston, MA, USA).

\section{Cell culture}

BREC cultures were established from fresh calf eyes and grown on fibronectin-coated flasks in DMEM with $10 \%$ fetal calf serum (FCS), $100 \mu \mathrm{g} / \mathrm{mL}$ EGS, $88 \mu \mathrm{g} / \mathrm{mL}$ heparin, and antibiotic-antimycotic solution (Sigma) under $5 \% \mathrm{CO}_{2}$, as described previously (Sone et al. 2000). Passages 2-10 were used for all experiments. Serum deprivation $(0.5 \%$ FCS $)$ was performed on all cultures for $16 \mathrm{~h}$ prior to the addition of growth factor, as described previously (Sone et al. 2000). Treatment of BREC cultures with the inhibitors PD98059, GF109203X, wortmannin or LY294002 involved the addition of each inhibitor for $30 \mathrm{~min}$ prior to the addition of IGF-1. After the addition of IGF-1, the cultures were incubated with both the inhibitor and the growth factor for the designated time interval. The concentrations selected for each inhibitor were based on prior experiments (Sone et al. 2000; DeBosch et al. 2001), as well as published reports (Vlahos et al. 1994; Alessi et al. 1995; Tsakiridis et al. 1995; Xia et al. 1996).

The effects of the various inhibitors on cell viability was assessed at the end of each experimental period by visual inspection and comparison of the morphology of cells treated with and without the inhibitor. In addition, possible cytotoxicity of PD98059 was tested using an MTT-based cell toxicity assay (Sigma) on BREC cultures in the presence of $100 \mu \mathrm{M}$ of the inhibitor for a 24-h period. The lack of toxicity of maximum concentrations of GF109203X, wortmannin and LY294002 on the BREC cultures for up to $24 \mathrm{~h}$ was previously confirmed by the same assay (DeBosch et al. 2001). The MTT assay was performed according to the manufacturer's instructions.

\section{Glucose transport studies}

Glucose transport assays were performed using $\left[{ }^{3} \mathrm{H}\right] 2 \mathrm{DG}$ (spec. activity of $25 \mathrm{Ci} / \mathrm{mmol}$ ), according methods described in detail previously (DeBosch et al. 2001) after treatment of serum-deprived, semiconfluent BREC cultures with or without IGF-1 $(25 \mathrm{ng} / \mathrm{mL})$ for $24 \mathrm{~h}$ in the presence and absence of the MEK1 inhibitor, PD98059 $(100 \mu \mathrm{M})$. The $25-\mathrm{ng} / \mathrm{mL}$ dose of IGF-1 and the incubation period 
were chosen because stimulation of BREC glucose transport by IGF-1 was previously found to be maximum at that dose and that duration (DeBosch et al. 2001). Results were expressed in pmol $\left[{ }^{3} \mathrm{H}\right] 2 \mathrm{DG}$ per mg protein per min. For each experiment, $n=4-5$ cultures in each group.

\section{Western blotting}

Western blotting analysis for phosphorylated and total ERK was performed on $10-\mu \mathrm{g}$ aliquots of solubilized BREC protein harvested from semiconfluent cultures that had been treated with and without IGF-1 $(25 \mathrm{ng} / \mathrm{mL})$ for various time points. Immunoblotting was performed with primary anti-phospho specific and anti-total ERK antisera, both at concentrations of $1: 1000$, using methods described previously (Sone et al. 2000).

\section{Kinase activity assays}

MAP kinase

MAP kinase activity was measured on semiconfluent, serumdeprived BREC cultures treated with and without IGF-1 $(25 \mathrm{ng} / \mathrm{mL})$ in the presence and absence of various inhibitors (PD98059, $100 \mu \mathrm{M}$; LY294002, $20 \mu \mathrm{M}$; GF109203X, $5 \mu \mathrm{M}$ ) for $15 \mathrm{~min}$ at $37^{\circ} \mathrm{C}$, using a p44/p42 MAP kinase assay kit from Cell Signaling Technologies. The assay was performed according to the manufacturer's instructions.

\section{Protein kinase $C$}

PKC activity was measured by an in vitro assay on permeabilized BREC cultures according to the methods of Heasley and Johnson (Heasley and Johnson 1989), with modifications as described previously (DeBosch et al. 2001). Serum-deprived BREC cultures were treated with and without the MEK1 inhibitor PD98059 $(100 \mu \mathrm{M})$ for $30 \mathrm{~min}$, followed by treatment with or without IGF-1 $(25 \mathrm{ng} / \mathrm{mL})$ for $15 \mathrm{~min}$. Previous time-course studies demonstrated that IGF-1 stimulated PKC activity optimally at $15 \mathrm{~min}$ (data not shown) and this increase in activity was completely abolished by pre-treatment with the PKC-selective inhibitor, GF109203X (DeBosch et al. 2001). After incubation, the cells were washed once with DMEM and $20 \mathrm{mM}$ HEPES ( $\mathrm{pH} 7.4$ ), and incubated in a salt solution containing $25 \mu \mathrm{g} / \mathrm{mL}$ of purified digitonin (Calbiochem), $100 \mu \mathrm{M}$ ATP, $14 \mu \mathrm{Ci} / \mathrm{mL}$ of $\left[\gamma-{ }^{32} \mathrm{P}\right]$ ATP (approximately $400 \mathrm{cpm} / \mathrm{pmol}$ or $1.8 \times 10^{5} \mu \mathrm{Ci} / \mathrm{pmol}$ ) and $300 \mu \mathrm{M}$ of the specific PKC substrate KRTLRR (Bachem, Torrance, CA, USA) for $15 \mathrm{~min}$ at $30^{\circ} \mathrm{C}$. At the end of the incubation, $45-\mu \mathrm{L}$ aliquots of the reaction mixture were spotted on Whatman P-81 phosphocellulose discs (Fisher Scientific, Pittsburg, PA, USA) and air-dried for at least $10 \mathrm{~min}$. The discs were then washed three times in $75 \mathrm{~mm}$ phosphoric acid, followed by one wash in sodium phosphate (pH 7.5). Radioactivity on the filters was measured by scintillation counting, and the activity was normalized for protein concentrations measured in parallel cultures under each experimental condition.

\section{PI3 kinase}

A PI3 kinase activity assay was performed on semiconfluent BREC cultures after overnight serum deprivation and treatment with or without IGF-1 $(25 \mathrm{ng} / \mathrm{mL})$ at $37^{\circ} \mathrm{C}$ for $10 \mathrm{~min}$ in the presence or absence of PD98059 $(100 \mu \mathrm{M})$, according to the methods of Tsakiridis et al. (1995), with modifications as described previously (DeBosch et al. 2001). Briefly, immunoprecipitation for IRS-1 was performed on 500- $\mu \mathrm{g}$ aliquots of the treated and control cultures with $2 \mu \mathrm{g}$ of anti-IRS-1 antibody (Upstate Biotechnologies Inc., Lake Placid, NY, USA), followed by precipitation with $60 \mu \mathrm{L}$ of a $25 \%$ Protein A agarose slurry. The immunoprecipitates were washed, and the kinase reaction was initiated by the addition of $26 \mu \mathrm{L}$ of a solution containing $13.8 \mathrm{~mm}$ $\mathrm{MgCl}_{2}, 0.156 \mathrm{~mm}$ ATP, $15 \mu \mathrm{Ci}\left[\gamma_{-}{ }^{32} \mathrm{P}\right] \mathrm{ATP}(3000 \mathrm{Ci} / \mathrm{mmol})$ and $40 \mu \mathrm{g}$ of L- $\alpha$-phosphatidylinositol (Avanti Polar Lipids, Alabaster, AL, USA). The reaction was terminated by the addition of $20 \mu \mathrm{L}$ of $1 \mathrm{M} \mathrm{HCl}$, and lipids were extracted with $160 \mu \mathrm{L}$ of $\mathrm{CHCl}_{3}: \mathrm{MeOH}(1: 1)$. Aliquots of the aqueous phase were loaded on an oxaloacetate-coated HPTLC silica glass plate (EM Science, Cincinnati, OH, USA), and a 50- $\mu$ g aliquot of phosphatidylinositol-3 phosphate (PI3P) standard (Matreya Inc., Pleasant Gap, PA, USA) suspended in $\mathrm{CHCl}_{3}$ was loaded for comparison. Lipids were separated by chromatography in $\mathrm{CHCl}_{3}: \mathrm{MeOH}$ : $\mathrm{H}_{2} \mathrm{O}: \mathrm{NH}_{4} \mathrm{OH}$ at a $60: 47: 11.6: 2$ ratio, and after air drying, the plate was exposed to autoradiographic film $24 \mathrm{~h}$ at room temperature $\left(25^{\circ} \mathrm{C}\right)$. The PI3P standard was visualized by ultraviolet light after treating the TLC plate with primulin-acetone, and quantification of the labeled reaction products was performed by scintillation counting of bands excised from the TLC plates.

\section{Statistical analysis}

Each experiment was repeated between two and four times with comparable results. The 2DG uptake assay was performed on either four or five cultures in each treatment group, and the results were expressed as mean $\pm \mathrm{SE}$. Analysis of data from the 2DG uptake assays, PKC activity assays and MAP kinase activity assays between the control and the various treatment groups was performed using either Student's $t$-test (MAP kinase activity) or analysis of variance (ANOvA, for 2DG uptake assay and PKC assay) with use of a post-hoc Bonferroni-Dunn correction for multiple variables. $p<0.05$ was considered significant for all experiments.

\section{Results}

\section{IGF-1 stimulates ERK phosphorylation and MAP kinase activity}

Treatment of BREC with IGF-1 (25 ng/mL, $3.3 \mathrm{~nm})$ led to rapid phosphorylation of ERK1 and ERK2, with a maximal increase at $15 \mathrm{~min}$ (Fig. 1a, upper panel, and Fig. 1b). Abundance of total ERK1/ERK2 did not change (Fig. 1a, lower panel). ERK1/ERK2 phosphorylation returned towards baseline at approximately $45 \mathrm{~min}$ (Figs 1a and b). This increase in phosphorylation correlated with increased MAP kinase activity, as assessed by measurement of the in vitro phosphorylation of an ERK substrate, ELK-1, in IGF-1-treated BREC cultures (Figs 1c and d). The increase in enzyme activity suggested that IGF-1 was stimulating dual phosphorylation of Thr183 and Tyr185 of ERK, resulting in full activation of the kinase (Seger and Krebs 1995). 
Fig. 1 IGF-1 Stimulates MAP Kinase in BREC Cultures. (a) Western blotting for phosphorylated ( $p E R K$ ) and total ERK was performed on primary BREC cultures treated with IGF-1 $(25 \mathrm{ng} / \mathrm{mL})$ for the times indicated. (b) Densitometric analysis of ratio of pERK to total ERK (tERK) for a representative western blot, pictured in (a). Western blotting analysis was repeated twice with identical results. (c) Representative results from an MAP kinase assay, which detects the ability of activated MAP kinase to phosphorylate ELK-1. (d) Densitometric analysis of the results of three separate MAP kinase activity assays of control and IGF-1-treated BREC cultures, expressed as percentage of control. ${ }^{*} p<0.02$, compared with control cultures.

MAP kinase activation is required for IGF-1 enhancement of BREC glucose transport

As reported previously (DeBosch et al. 2001), IGF-1 stimulated an approximate doubling of $\left[{ }^{3} \mathrm{H}\right] 2 \mathrm{DG}$ uptake in semiconfluent BREC cultures (Fig. 2). This increase was previously shown to reflect IGF-1 actions on glucose transport per se, rather than increased glucose metabolism, because treatment of BREC cultures with an identical concentration of IGF-1 produced a comparable increase in

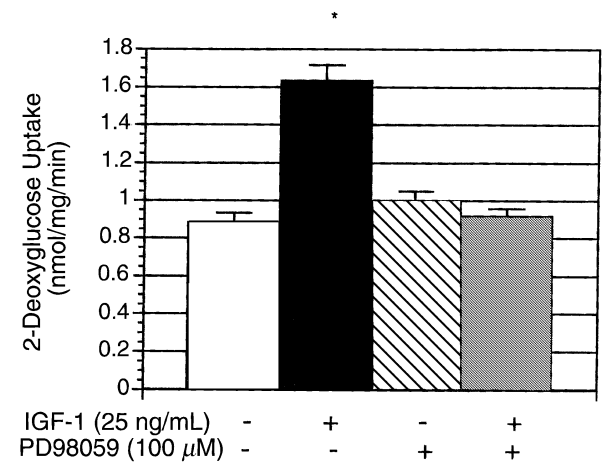

Fig. 2 Effects of MAP kinase inhibition on BREC glucose transport. Representative $\left[{ }^{3} \mathrm{H}\right] 2 \mathrm{DG}$ uptake assay of control and IGF-1 treated BREC cultures in the presence (+) and absence (-) of the selective MEK inhibitor, PD98059 (100 $\mu \mathrm{m})$. Cultures were treated with $25 \mathrm{ng} / \mathrm{mL}$ for $24 \mathrm{~h}$, a concentration and duration previously demonstrated to result in maximal glucose uptake in BREC cultures (DeBosch et al. 2001). $n=4$ cultures per treatment group; ${ }^{\star} p<0.0001$, compared with controls. All others not statistically significant compared with controls. (b)

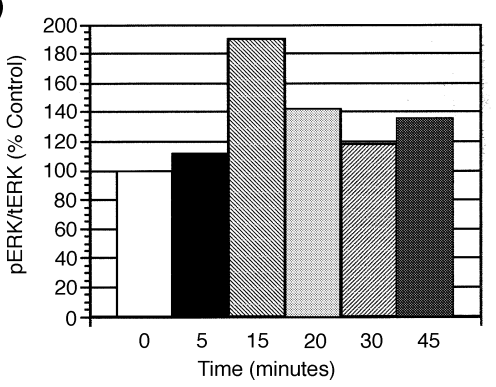

(d)

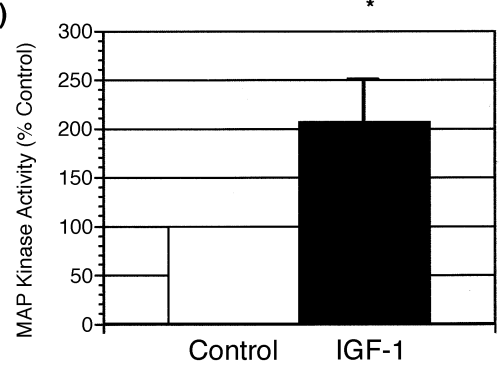

the uptake of 3-O-methylglucose, a glucose analog that is transported into, but not metabolized by, retinal endothelial cells (DeBosch et al. 2001). IGF-1 enhancement of BREC glucose transport was completely abrogated with treatment with the selective MEK1 inhibitor, PD98059 (100 $\mu \mathrm{M}$, Fig. 2) (Alessi et al. 1995), and treatment of BREC cultures with PD98059 alone had no effect (Fig. 2). Cells treated with $100 \mu \mathrm{M}$ PD98059 for $24 \mathrm{~h}$ were morphologically indistinguishable from untreated cells, and cell viability, as assessed by MTT staining of the cells, was no different between PD98059-treated and untreated cultures (data not shown).

PKC activation by IGF-1 is dependent on MAP kinase The dependence of IGF-1-stimulated enhancement of retinal endothelial cell glucose transport on $\mathrm{PKC}$, and in particular, PKC- $\beta$, has been demonstrated previously (DeBosch et al. 2001). In order to clarify the interaction between PKC and MAP kinase, in situ PKC assays were performed on permeabilized BREC cultures without and with pre-treatment by the MEK1 inhibitor PD98059 $(100 \mu \mathrm{M})$. Inhibition of MEK1 abolished PKC activity in IGF-1-treated cultures (Fig. 3). Exposure of the cultures to PD98059 alone did not affect PKC activity (data not shown).

PI3 kinase activation is not dependent on MAP kinase In order to understand the relationship between stimulation of MAP kinase activity and stimulation of PI3 kinase by IGF-1, PI3 kinase assays were performed on IRS-1 immunoprecipitates of BREC cultures pretreated with PD98059 $(100 \mu \mathrm{M})$ and stimulated with IGF-1 $(25 \mathrm{ng} / \mathrm{mL}$, 10 min). As shown by Fig. 4, inhibition of MAP kinase 


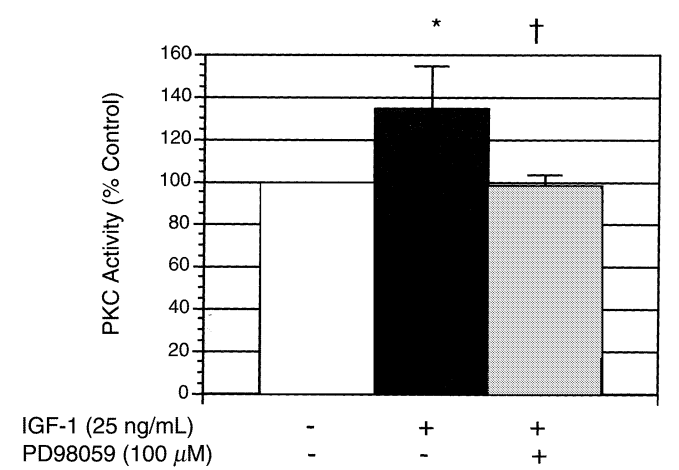

Fig. 3 Effects of PKC and MAP kinase inhibition on IGF-1-stimulated PKC activity in BREC cultures. Semi-confluent BREC cultures were incubated with and without IGF-1 $(25 \mathrm{ng} / \mathrm{mL})$ for $15 \mathrm{~min}$ in the presence (+) and absence (-) of the selective MEK inhibitor, PD98059 $(100 \mu \mathrm{M})$, and an in vitro assay for PKC was performed on the permeabilized cells as described in Materials and methods. The results are expressed as a percentage of control PKC activity and represent the mean $\pm \mathrm{SE}$ of three independent experiments on separate sets of cultures with duplicate samples in each experiment. ${ }^{*} p<0.02$ compared with control; $\uparrow p$ is not significant compared with control.

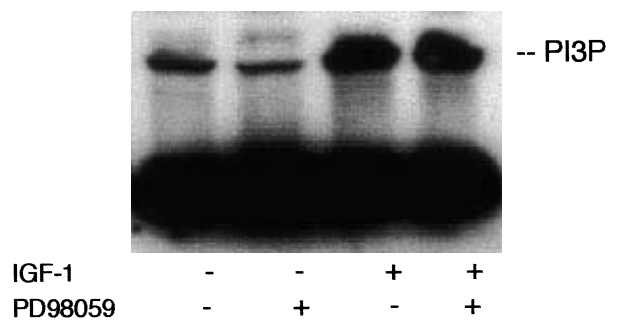

Fig. 4 Effects of PI3 kinase, PKC and MAP kinase inhibition on IGF1-stimulated $\mathrm{PI} 3$ kinase activity in BREC cultures. Semi-confluent BREC cultures were incubated with and without IGF-1 $(25 \mathrm{ng} / \mathrm{mL})$ for $10 \mathrm{~min}$ in the presence $(+)$ and absence $(-)$ of the MEK inhibitor, PD98059 $(100 \mu \mathrm{M})$, and PI3 kinase assays were performed as described in Materials and methods. Results shown are representative of PI3 kinase assays which were repeated twice with identical results.

activity with PD98059 had no effect on IGF-1-mediated increases in PI3 kinase activity. These data were similar to those reported previously (DeBosch et al. 2001), which demonstrated that inhibition of PKC with GF109203X had no effect on IGF-1-stimulated PI3 kinase activity in BREC cultures.

\section{MAP kinase activation by IGF-1 is not dependent on activation by PI3 kinase or PKC}

The experiments described above that investigated the effects of inhibition of MAP kinase on either PKC or PI3 kinase activity assays in IGF-1-treated BREC cultures suggested that: (i) activation of PKC by IGF-1 in BREC cultures was dependent on MAP kinase; and (ii) in contrast, activation of
PI3 kinase by IGF-1 was independent of MAP kinase, either because PI3 kinase was 'upstream' from MAP kinase, i.e. the activation of PI3 kinase by IGF-1 preceded IGF-1 effects on MAP kinase, or because IGF-1 stimulation of MAP kinase within retinal endothelial cells - and IGF-1 effects on MAP kinase-mediated changes in glucose transport - occurred without the involvement of PI3 kinase. In order to test these possibilities, Western blotting for phosphorylated ERK was performed on control and IGF-1-treated BREC cultures after pre-treatment with and without inhibitors to MAP kinase (PD98059, $100 \mu \mathrm{M}$ ), PI3 kinase (wortmannin, $100 \mathrm{nM}$ ) and PKC (GF109203X, $5 \mu \mathrm{M}$ ). In preliminary experiments, exposure of BREC cultures to wortmannin alone (100 nM) resulted in significant suppression of ERK phosphorylation in the absence of IGF-1 (data not shown), which made assessment of the effects of PI3 kinase inhibition on IGF-1 stimulated ERK phosphorylation difficult to interpret. Therefore, in these experiments, the effects of PI3 kinase inhibition on ERK phosphorylation were studied using LY294002 $(20 \mu \mathrm{M})$, a highly selective PI3 kinase inhibitor that is structurally unrelated to wortmannin (Vlahos et al. 1994). As can be seen in Fig. 5(a), IGF-1 increased ERK phosphorylation in BREC cultures and was completely inhibited by pre-treatment with the MEK1 inhibitor, PD98059. In contrast, PI3 kinase inhibition with LY294002 $(20 \mu \mathrm{M})$ and PKC inhibition with GF109203X (5 $\mu \mathrm{M})$ had no effect on IGF-1-stimulated ERK phosphorylation (Fig. 5a). Stimulation of BREC cultures by IGF-1 and treatment of these cultures with the various inhibitors had no effect on the abundance of total ERK (Fig. 5a). Pre-treatment of cultures with PD98059, LY294002 and GF109203X alone had no

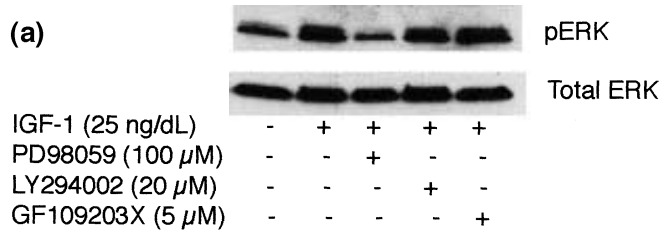

(b)

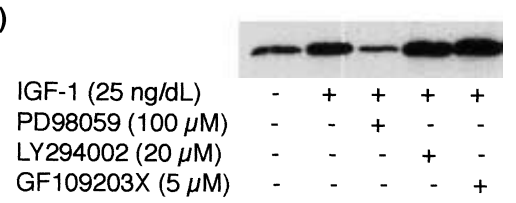

Fig. 5 Effects of PI3 kinase, PKC and MAP kinase inhibition on IGF1-stimulated ERK phophorylation and MAP kinase activity in BREC cultures. (a) Phosphorylated (pERK) and total ERK western blots of BREC cultures without and with treatment with IGF-1 (25 ng/mL for $15 \mathrm{~min}$ ) in the presence of the various inhibitors. (b) MAP kinase activity assay of BREC cultures without and with treatment with IGF-1 (25 $\mathrm{ng} / \mathrm{mL}$ for $15 \mathrm{~min}$ ) in the presence of the various inhibitors. Results are representative of assays repeated twice with identical results. 
effect on either phosphorylated or total ERK levels (data not shown). These observations were supported by in vitro MAP kinase activity assays on cultures treated with PD98059

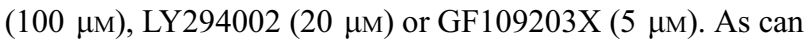
be seen in Fig. 5(b), the increase in MAP kinase activity seen with IGF-1 stimulation was completely abolished with treatment with PD98059; in contrast however the increase in MAP kinase activity after IGF-1 stimulation was unaffected by either LY294002 or GF109203X.

\section{Discussion}

We have previously shown that IGF-1 enhances glucose transport and proliferation in primary cultures of retinal endothelial cells and does so in a PKC- and PI3 kinasedependent manner (DeBosch et al. 2001). In the current study, we have extended these investigations to clarify the role of MAP kinase in IGF-1-mediated changes in glucose transport. Herein, we report that ERK phosphorylation and MAP kinase activity are increased in IGF-1-treated BREC cultures (Fig. 1), and selective inhibition of MAP kinase activity in these cultures abolishes the stimulation of glucose transport by IGF-1 (Fig. 2).

Stimulation of ERK phosphorylation by IGF-1 is an acute event in cultured retinal endothelial cells, reaching a maximum within approximately $15 \mathrm{~min}$ and returning towards baseline within 45 min (Fig. 1a). This stimulation requires the upstream involvement of MEK1/MEK2, because pharmacological inhibition of MEK1/MEK2 with PD98059 abrogates the ability of IGF-1 to phosphorylate ERK1 and ERK2 (Fig. 5a) and abolishes their ability to phosphorylate a specific substrate, ELK-1 (Fig. 5b). In contrast to the relatively transient stimulation of MAP kinase by IGF-1, the consequences of IGF-1-mediated activation MAP kinase on retinal endothelial cell glucose transport is a relatively slow process, reaching a maximum within $24 \mathrm{~h}$ after exposure (DeBosch et al. 2001). Nonetheless, inhibition of this transient activation of MAP kinase by pre-treatment with PD98059 completely abolishes the ability of IGF-1 to enhance BREC glucose transport (Fig. 2). These and earlier studies (DeBosch et al. 2001) demonstrate that MAP kinase, PKC and PI3 kinase all participate in IGF-1-mediated stimulation of glucose uptake in BREC. Presumably, binding of the IGF-1 to its receptor initiates signal transduction pathways involving PKC, PI3 kinase and MAP kinase within minutes, and stimulation of these pathways in turn lead to cellular - and perhaps nuclear - events that culminate in increased glucose transport within hours. Although activation of MAP kinase may involve phosphorylation of nuclear regulatory proteins such as c-Jun and c-Fos, the actions of IGF-1 on enhancement of BREC glucose transport do not appear to involve new synthesis of GLUT1, because total cellular
GLUT1 protein and mRNA do not change following exposure of BREC to concentrations of IGF-1 known to stimulate transport $(25 \mathrm{ng} / \mathrm{mL})$ (DeBosch et al. 2001).

The increase in glucose transport in the absence of an increase in total cellular GLUT1 abundance reportedly earlier in IGF-1-treated BREC cultures (DeBosch et al. 2001) suggests that IGF-1 actions on glucose transport occurs through translocation of cytosolic GLUT1 to the plasma membrane. In this context, the situation is perhaps similar to the stimulation of glucose transport in these cells by VEGF, in which enhancement of glucose transport occurs in the absence of an increase in GLUT1 protein or transcript (Sone et al. 2000). In the case of VEGF, stimulation by this growth factor results in increased abundance of GLUT1 at the plasma membrane (Sone et al. 2000) and suggests that VEGF-stimulated glucose uptake in BREC occurs via translocation of GLUT1 from intracellular compartments.

The current study also documents the relationship between PKC, PI3 kinase and MAP kinase in IGF-1-stimulated glucose transport in retinal endothelial cells. As has been previously demonstrated, activation of PKC in these cells is dependent on prior activation of PI3 kinase, because pretreatment with an inhibitor of PI3 kinase, wortmannin, abolishes the ability of IGF-1 to stimulate PKC activity (DeBosch et al. 2001). In a similar manner, IGF-1 stimulation of PKC in BREC is also dependent on increased MAP kinase activity, as pre-treatment of cultures with the MEK1 inhibitor, PD98059, abolishes IGF-1-mediated increases in PKC activity (Fig. 3). In contrast, activation of MAP kinase by IGF-1 is independent of its actions on PI3 kinase: inhibition of MAP kinase with PD98059 does not affect PI3 kinase activity (Fig. 4), and conversely, inhibition of PI3 kinase has no effect on the phosphorylation of ERK1 and ERK2, and on the stimulation of MAP kinase activity by IGF-1 (Figs 5a and b). The independence of PI3 kinase signaling from MAP kinase pathway activity in IGF1-treated BREC cultures is similar to the effects of IGF-1 on PI3 kinase and MAP kinase activity in other cells types. For example, the increased PI3 kinase activity, as well as p85 and akt phosphorylation, which is responsible for the increased proliferative capacity of cultured satellite cells isolated from transgenic mice overexpressing skeletal muscle IGF-1, is unaffected by treatment with the MEK inhibitor PD98059, and conversely treatment of these cells with the PI3 kinase inhibitor LY294002 does not inhibit increased MAP kinase phosphorylation by IGF-1 (Chakravarthy et al. 2000). Similarly, IGF-1-stimulates mitogenesis in $\mathrm{C} 2 \mathrm{C} 12$ muscle cells via activation of PI3 kinase and independent of MAP kinase activity (Milasincic et al. 1996). Taken together, the experiments of the current study suggest that PKC may be activated by MAP kinase and PI3 kinase through separate, independent pathways in retinal endothelial cells. Nonetheless, these data also suggest that stimulation of PKC by IGF-1 
through either pathway alone is not sufficient to enhance glucose transport, because inhibition of PI3 kinase, MAP kinase and PKC separately may block the ability of IGF-1 to increase glucose transport in BREC cultures (DeBosch et al. 2001 and this study).

In summary, the present study demonstrates that IGF-1 stimulation of the MAP kinase pathway participates in the enhancement of BREC glucose transport by this growth factor. This pathway is required for both activation of PKC and stimulation of glucose transport, because inhibition of MAP kinase abrogates the actions of IGF-1 on both processes. Nonetheless, IGF-1-mediated activation of MAP kinase alone is not sufficient to stimulate glucose transport in these cells, as inhibition of PI3 kinase signaling, which is independent from that of MAP kinase, can inhibit glucose uptake. Elucidation of the signal transduction pathways used by IGF-1 in regulating retinal endothelial cell glucose transport demonstrates the complexity of the actions of this growth factor in the retina in states of health and disease.

\section{Acknowledgements}

The authors are indebted to Drs C. Carter-Su, F. C. Brosius, and E. L. Feldman (University of Michigan) for many helpful discussions. This work was supported by National Institutes of Health grant K08 EY000369 (AKK) and the Juvenile Diabetes Research Foundation (AKK). BJD was supported by a Honors Project stipend from the University of Michigan. AKK is supported by the JDRF Center for Complications in Diabetes and is supported in part by National Institutes of Health Grant RPO60DK-20572, which supports the Michigan Diabetes Research \& Training Center.

\section{References}

Alessi D. R., Cuenda A., Cohen P., Dudley D. T. and Saltiel A. R. (1995) PD 098059 is a specific inhibitor of the activation of mitogenactivated protein kinase in vitro and in vivo. J. Biol. Chem 270, 27489-27494.

Chakravarthy M. V., Abraha T. W., Schwartz R. J., Fiorotto M. L. and Booth F. W. (2000) Insulin-like Growth Factor-I Extends in Vitro Replicative Life Span of Skeletal Muscle Satellite Cells by Enhancing G1/S Cell Cycle Progression via the Activation of Phosphatidylinositol 3'-Kinase/Akt Signaling Pathway. J. Biol. Chem. 275, 35942-35952.

Cunha-Vaz J. G. (1976) The blood-retinal barriers. Doc. Ophthal. 41, 287-327.

DeBosch B. J., Deo B. K., Baur E., Deo B. K., Hiraoka M. and Kumagai A. K. (2001) Effects of Insulin-like growth factor-1 on retinal endothelial cell glucose transport and proliferation. J. Neurochem. 77, 1157-1167.

English J., Pearson G., Wilsbacher J., Swantek J., Karandikar M., Xu S. and Cobb M. H. (1999) New insights into the control of MAP kinase pathways. Exp. Cell Res. 253, 255-270.

Heasley L. E. and Johnson G. L. (1989) Regulation of protein kinase C by nerve growth factor, epidermal growth factor, and phorbol esters in PC12 pheochromocytoma cells. J. Biol. Chem 264, 8646-8652.

Knott R. M., Robertson M., Muckersie E. and Forrester J. V. (1996) Glucose-mediated regulation of GLUT-1 and GLUT-3 mRNA in human retinal endothelial cells. Biochem. Soc. Trans. 24, $216 \mathrm{~S}$.

Kumagai A. K. (1999) Glucose transport in brain and retina: implications in the management and complications of diabetes. Diab. Metab. Res. Rev. 15, 261-273.

Mandarino L. J., Finlayson J. and Hassell J. R. (1994) High glucose downregulates glucose transport activity in retinal capillary pericytes but not endothelial cells. Invest. Ophthalmol. Vis. Sci. 35, 964-972.

Mantych G. J., Hageman G. S. and Devaskar S. U. (1993) Characterization of glucose transporter isoforms in the adult and developing human eye. Endocrinology 133, 600-607.

Milasincic D., Calera M., Farmer S. and Pilch P. F. (1996) Stimulation of $\mathrm{C} 2 \mathrm{C} 12$ myoblast growth by basic fibroblast growth factor and insulin-like growth factor 1 can occur via mitogen-activated protein kinase-dependent and -independent pathways. Mol. Cell. Biol. 16, 5964-5973.

Robinson G. S. and Aiello L. P. (1998) Angiogenic factors in diabetic ocular disease: mechanisms of today, therapies for tomorrow. Int. Ophthalmol Clin. 38, 89-102.

Seger R. and Krebs E. G. (1995) The MAPK signaling cascade. FASEB J. 9, 726-735.

Sone H., Deo B. K. and Kumagai A. K. (2000) Enhancement of glucose transport by vascular endothelial growth factor in retinal endothelial cells. Invest. Ophthalmol. Vis. Sci. 41, 1876-1884.

Takagi H., King G. L. and Aiello L. P. (1998) Hypoxia upregulates glucose transport activity through an adenosine-mediated increase of GLUT1 expression in retinal capillary endothelial cells. Diabetes 47, 1480-1488.

Takata K., Hirano H. and Kasahara M. (1997) Transport of glucose across the blood-tissue barriers. Int. Rev. Cytol. 172, 1-53.

Tsakiridis T., McDowell H. E., Walker T., Downes C. P., Hundal H. S., Vranic M. and Klip A. (1995) Multiple roles of phosphatidylinositol 3-kinase in regulation of glucose transport, amino acid transport, and glucose transporters in L6 skeletal muscle cells. Endocrinology 136, 4315-4322.

Vlahos C. J., Matter W. F., Hui K. Y. and Brown R. F. (1994) A specific inhibitor of phosphatidylinositol 3-kinase, 2-(4-morpholinyl)8-phenyl-4H-1-benzopyran-4-one (LY294002). J. Biol. Chem. 269, 5241-5248.

Xia P., Aiello L. P., Ishii H., Jiang Z. Y., Park D. J., Robinson G. S., Takagi H., Newsome W. P., Jirousek M. R. and King G. L. (1996) Characterization of vascular endothelial growth factor's effect on the activation of protein kinase $\mathrm{C}$, its isoforms, and endothelial cell growth. J. Clin. Invest. 98, 2018-2026. 